\title{
The microstructure network and thermoelectric properties of bulk $(\mathrm{Bi}, \mathrm{Sb})_{2} \mathrm{Te}_{3}$
}

\author{
Wenjie Xie, ${ }^{1,2}$ Dale A. Hitchcock, ${ }^{1}$ Hye J. Kang, ${ }^{1, a)}$ Jian He, ${ }^{1}$ Xinfeng Tang, ${ }^{2, b)}$ \\ Mark Laver, ${ }^{3,4,5}$ and Boualem Hammouda ${ }^{6}$ \\ ${ }^{1}$ Department of Physics and Astronomy, Clemson University, Clemson, South Carolina 29634, USA \\ ${ }^{2}$ State Key Laboratory of Advanced Technology for Materials Synthesis and Processing, Wuhan University \\ of Technology, Wuhan 430070, China \\ ${ }^{3}$ Department of Physics, Technical University of Denmark, DK-2800 Kgs. Lyngby, Denmark \\ ${ }^{4}$ Nano-Science Center, Niels Bohr Institute, University of Copenhagen, DK-2100 København, Denmark \\ ${ }^{5}$ Laboratory for Neutron Scattering, Paul Scherrer Institut, CH-5232 Villigen PSI, Switzerland \\ ${ }^{6}$ NIST Center for Neutron Research, National Institute of Standards and Technology, Gaithersburg, \\ Maryland 20899, USA
}

(Received 18 June 2012; accepted 28 August 2012; published online 12 September 2012)

We report small-angle neutron scattering studies on the microstructure network in bulk $(\mathrm{Bi}, \mathrm{Sb})_{2} \mathrm{Te}_{3}$ synthesized by the melt-spinning (MS) and the spark-plasma-sintering (SPS) process. We find that rough interfaces of multiscale microstructures generated by the MS are responsible for the large reduction of both lattice thermal conductivity and electrical conductivity. Our study also finds that subsequent SPS forms a microstructure network of $\sim 10 \mathrm{~nm}$ thick lamellae and smooth interfaces between them. This nanoscale microstructure network with smooth interfaces increases electrical conductivity while keeping a low thermal conductivity, making it an ideal microstructure for high thermoelectric efficiency. (C) 2012 American Institute of Physics. [http://dx.doi.org/10.1063/1.4752110]

In thermoelectrics, much effort has been made to reduce the lattice thermal conductivity without degrading the electrical conductivity to improve thermoelectric efficiency. Heavily doped semiconductors have been good candidates for thermoelectric materials. This is due to low carrier thermal conductivity, an adequate Seebeck coefficient and acceptable electrical conductivity upon doping. The key task is to find ways to lower the lattice thermal conductivity, which is the majority contribution to thermal conductivity in semiconductors, without degrading electrical conductivity.

Studies have shown that nanostructures significantly reduce the lattice thermal conductivity. ${ }^{1-7}$ Studies on the thermal conduction mechanism in superlattices show that the introduction of many interfaces scatters phonons much more effectively than electrons. ${ }^{4,7,8}$ These studies also suggest that the periodicity of superlattices is not a necessary condition for the reduction of the lattice thermal conductivity. Naturally, nanocomposites have drawn attention as high performance thermoelectric materials in the bulk form, which is a more practical form for many applications. Nanoparticles in the nanocomposites have various types of distributions such as particle sizes, size distributions, and random spatial distribution. Model calculations show that the thermal conductivity depends mainly on the interface density associated with the nanostructures. $4,7,8$

The role of nanostructures to thermoelectric properties has been studied in the $\mathrm{Bi}_{2} \mathrm{Te}_{3}$ alloy. $\mathrm{Bi}_{2} \mathrm{Te}_{3}$ alloy is a good basis for thermoelectric materials, since it has a high electrical conductivity and a low thermal conductivity. Doping $\mathrm{Bi}_{2} \mathrm{Te}_{3}$ with holes, $\mathrm{Bi}_{0.5} \mathrm{Sb}_{1.5} \mathrm{Te}_{3}$ is found to have its highest $Z T \approx 1\left(Z T=\frac{\sigma \alpha^{2}}{\kappa} T\right.$, where $\sigma, \alpha, T$, and $\kappa$ are electrical con-

\footnotetext{
a) Author to whom correspondence should be addressed. Electronic mail: hkang70@clemson.edu.

b)Electronic mail: tangxf@whut.eud.cn.
}

ductivity, Seebeck coefficient, absolute temperature, and thermal conductivity, respectively. ${ }^{9}$ ) at room temperature. ${ }^{10}$ Recently, two groups synthesized $(\mathrm{Bi}, \mathrm{Sb})_{2} \mathrm{Te}_{3}$ nanocomposites using a ball-milled ingot followed by hot pressing and using a melt-spinning (MS) elements followed by the sparkplasma-sintering (SPS) process. This achieved a maximum ZT of 1.4 at $373 \mathrm{~K}$ from one group ${ }^{11}$ and 1.5 at $390 \mathrm{~K}$ from the other. ${ }^{12}$ Both groups concluded that the increase of $Z T$ comes from the reduction in the thermal conductivity due to the increase in phonon scattering at interfaces. Unfortunately, the scattering at interfaces can also lower the electrical conductivity, which is not desirable for the increase of thermoelectric efficiency. This is observed in our current study. Consequently, it is crucial to find ways of generating interfaces which enhance phonon scattering and at the same time reduce electron scattering. Our manuscript will show that the combined MS-SPS process is an excellent way to satisfy this condition.

We believe that the presence of interfaces alone cannot explain their significant impact on thermal conductivity and electrical conductivity, since in nanocomposites, the volume fraction of nanophase is only a few percent. In order to explain the large impact of a small percentage of nanostructures on both thermal conductivity and electrical conductivity, the connectivity of these nanostructures must be considered. Therefore, it is extremely important to study the nanostructure network to find the optimal conditions for a low thermal conductivity and a high electrical conductivity. In this manuscript, we report on small-angle neutron scattering studies of the microstructure network in bulk $(\mathrm{Bi}, \mathrm{Sb})_{2} \mathrm{Te}_{3}$ nanocomposites synthesized by the MS-SPS process. Our study also reports on the roles of the MS and SPS process on the network formation of microstructures.

The MS process generates a wide spectrum of multiscale microstructures from micron-sized to nanometer-sized 
structures. ${ }^{12}$ It is believed that these multiscale microstructures are good for reducing the lattice thermal conductivity. However, the specific roles of the MS and SPS on the generation of microstructures and a microstructure network that affect the thermoelectric properties have not been studied. To study the effect of the MS, we prepared a series of $\mathrm{Bi}_{0.48} \mathrm{Sb}_{1.52} \mathrm{Te}_{3}$ samples from zone-melted ingots (ZMingots). In a $0.06 \mathrm{MPa}$ argon atmosphere, ZM-ingots $\mathrm{Bi}_{0.48} \mathrm{Sb}_{1.52} \mathrm{Te}_{3}$ were melted and sputtered onto a watercooled copper roller rotating with speeds of 1000, 2000, 3000 , and $4000 \mathrm{rpm}$. The resulting MS ribbons were hand ground and subsequently SPS processed at $500{ }^{\circ} \mathrm{C}$. These samples are labeled as ZM-MSx-SPS500, $\mathrm{x}$ is the rotation speed. X-ray diffraction and energy-dispersive $\mathrm{X}$-ray spectroscopy show that all samples are in a single phase and the MS and SPS processes have little effect on the composition of the samples.

To study the microstructures, small-angle neutron scattering experiments were performed at the NG3 and NG7 instruments at the NIST Center for Neutron Research. Fig. 1 shows the scattering intensity $I(Q)$ versus momentum transfer $Q$ for the ZM-MSx-SPS500 samples as a function of rotation speed and the ZM-ingot. Small-angle scattering results from density fluctuations of the structures in the sample. The scattering is maximal at $Q \approx 2 \pi / d$, where $d$ is the characteristic lengthscale of the density fluctuations. No scattering would be observed in a homogeneous single crystal. The high $Q$ region $(Q d \gg 1)$ of the scattering from an inhomogeneity is called the Porod regime. The intensity $I(Q)$ in the Porod regime generally follows a power-law $I(Q)=\frac{A}{Q^{m}}$ $+b k g$,where $A, m$, and $b k g$ are the scale constant, the Porod exponent, and the incoherent background, respectively. For the ZM-ingot, $I(Q)$ closely follows the power-law with $m=3.86( \pm 0.01)$ between $Q_{\min }=0.001 \AA^{-1}$ and

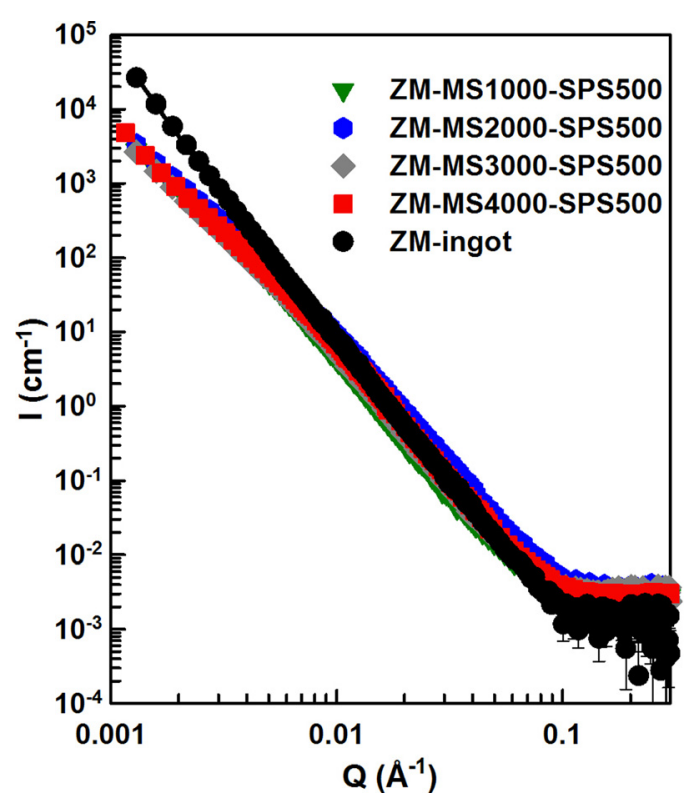

FIG. 1. Small-angle neutron scattering data of bulk ZM-MSx-SPS500 samples as a function of rotation speed during the MS. A ZM-ingot follows the power-law over the entire $Q$ range. All ZM-MSx-SPS500 samples deviate from the power-law at low $Q$. The solid line is the power-law fit to the ZM-ingot data. The statistical error bars are one standard deviation.
$Q_{\max }=0.1 \AA^{-1}$ (Fig. 1 and Table I). The Porod regime and power-law behavior extend down to the lowest $Q$ probed in our experiment, indicating that the ZM-ingot has microstructures that are polydisperse in size with the upper size much larger than $600 \mathrm{~nm}\left(2 \pi / Q_{\text {min }}\right)$. The Porod exponent $m$ probes the fractal dimension $D$ of the microstructural surface, specifically $m=6-D .^{13}$ For smooth interfaces, $D=2$ and $m=4$. Any roughness of the surface leads to $D>2$, with $m=3$ representing a very rough interface. $m=3.86$ being close to 4 in the ZM-ingot indicates that the microstructures in this sample have smooth interfaces. In contrast, the intensity for all ZM-MSx-SPS500 samples deviate from the power-law at low $Q$. This indicates the existence of a microstructure incorporating characteristic lengthscales smaller than $600 \mathrm{~nm}$. Additional information on size, shape, and network of this microstructure can be obtained by fitting the data using the generalized Guinier-Porod model. ${ }^{14} \mathrm{~A}$ detailed analysis will be described later in this paper with additional experimental data. The ZM-MSx-SPS500 samples still follow the power-law at high $Q$ with $m=3.78$ very similar to that seen in the ZM-ingot. This suggests that the distinct microstructures also have smooth interfaces. Data in Fig. 1 indicate that rotation speed values higher than $1000 \mathrm{rpm}$ in the MS process have no effect on the intensity. Higher speeds in the MS process are believed to generate more nanometer-sized structures. ${ }^{12}$ Thus, no change in the intensity for speeds above $1000 \mathrm{rpm}$ indicates no significant change in the generation of nanometer-sized particles in the bulk samples above a rotation speed of $1000 \mathrm{rpm}$.

Since our samples are prepared using two processes, we want to find the role of each process in the generation of microstructures and their network formation in the bulk samples. To study the role of the MS, we synthesized samples at rotation speeds of $1000,2000,3000$, and $4000 \mathrm{rpm}$ during the MS process. The MS ribbons were hand ground and cold pressed at $100 \mathrm{MPa}$. These samples are labeled as ZM-MSx, $\mathrm{x}$ represents the rotation speed. The small-angle neutron scattering result on the ZM-MSx samples is shown in Fig. 2(a). The same scattering behavior in all ZM-MSx samples indicates no effect of rotation speed above $1000 \mathrm{rpm}$ in the MS process, as expected from the data in Fig. 1. All ZM-MSx samples follow the power-law behavior just like the ZMingot in a wide $Q$ range, indicating that ZM-MSx samples also have microstructures that are polydisperse in size and random in spatial distribtution. This is consistent with a recent electron microscopy study showing that the MS process generates microstructures with random orientation and size. ${ }^{12}$ However, the ZM-MSx samples have $m=3.40$ $( \pm 0.01)$ (Table I). This substantial deviation of $m$ from 4 shows that the microstructures in the ZM-MSx samples have much rougher interfaces. Therefore, the main role of the MS is the generation of multiscale microstructures with very rough interfaces.

To study the role of the SPS, we chose the ZM-MS4000 samples (rotation speed of $4000 \mathrm{rpm}$ in the MS). They were SPS processed at different temperatures, $300{ }^{\circ} \mathrm{C}, 400{ }^{\circ} \mathrm{C}$, and $500^{\circ} \mathrm{C}$. These samples are labeled as ZM-MS4000-SPSy, y is the SPS temperature. The small-angle neutron scattering results from the ZM-MS4000-SPSy samples are shown in Fig. 2(b). The SPS processed sample at $300^{\circ} \mathrm{C}$ shows a 
TABLE I. Fitted parameters from the power-law and the generalized Guinier-Porod model in the ZM-MSx and ZM-MS4000-SPSy samples. The data are fitted to each model with least squares method. The models include instrument resolution effects. The statistical error is one standard deviation.

\begin{tabular}{|c|c|c|c|c|}
\hline Samples & Models & $m$ & $s$ & $R_{g}(\AA)$ \\
\hline ZM-ingot & Power law & $3.86( \pm 0.01)$ & $\ldots$ & $\ldots$ \\
\hline All ZM-MSx & Power law & $3.40( \pm 0.01)$ & $\ldots$ & $\ldots$ \\
\hline ZM-MS4000-SPS300 & Power law & $3.59( \pm 0.01)$ & $\ldots$ & $\ldots$ \\
\hline ZM-MS4000-SPS400 & Generalized Guinier-Porod & $3.82( \pm 0.01)$ & $2.35( \pm 0.01)$ & $51( \pm 1)$ \\
\hline ZM-MS4000-SPS500 & Generalized Guinier-Porod & $3.78( \pm 0.01)$ & $2.69( \pm 0.01)$ & $36( \pm 1)$ \\
\hline
\end{tabular}

power-law behavior with $m=3.59( \pm 0.01)$ (Table I). This indicates the SPS temperature of $300^{\circ} \mathrm{C}$ is not high enough to affect the microstructures in the bulk samples. However, an increase of $m$ compared to that of the ZM-MSx samples suggests that the SPS process makes the microstructures' surfaces smoother. At SPS temperatures higher than $300^{\circ} \mathrm{C}$, samples deviate from a power-law behavior at low $Q$, indicating that specific lengthscale microstructures are favored. To get a more quantitative analysis of the shape, network, and interface of microstructures, the data of ZM-MS4000SPSy samples are fitted with the generalized Guinier-Porod model. ${ }^{14}$ The standard Guinier plot provides the radius of gyration for compact objects from the slope of $\log (I(Q))$ vs $Q^{2}{ }^{15}$ The generalized Guinier-Porod plot provides the cross sectional radius of gyration (1) for elongated rod-like objects from the slope of $\log (Q I(Q))$ vs $Q^{2}$ and (2) for platelet-like objects from the slope of $\log \left(Q^{2} I(Q)\right)$ vs $Q^{2}$. ${ }^{16}$ The generalized Guinier-Porod model incorporates a degree of freedom in the exponent and thus obtains all such radii of gyration for the various compact, as well as elongated rod-like and platelike objects and in-between structures. The intensity in the generalized Guinier-Porod model is given by the two contributions: ${ }^{14}$

$$
\begin{aligned}
& I(Q)=\frac{G}{Q^{s}} \exp \left[\frac{-Q^{2} R_{g}{ }^{2}}{3-s}\right] \text { for } Q \leq Q_{1}, \\
& I(Q)=\frac{F}{Q^{m}} \text { for } Q \geq Q_{1} .
\end{aligned}
$$

Applying the continuity of the Guinier and Porod functions and their derivatives yields

$$
\begin{aligned}
Q_{1}= & \frac{1}{R_{g}} \sqrt{\frac{(m-s)(3-s)}{2}}, \\
F= & G \exp \left[\frac{-Q_{1}{ }^{2} R_{g}^{2}}{3-s}\right] Q_{1}^{(m-s)}=\frac{G}{R_{g}(m-s)} \\
& \times \exp \left[-\frac{(m-s)}{2}\right]\left(\frac{(m-s)(3-s)}{2}\right)^{\frac{(m-s)}{2}},
\end{aligned}
$$

$s$ is the Guinier exponent at low $Q$ associated with the shape and network of the microstructures, $m$ is the Porod exponent at high $Q$ associated with interface roughness, $R_{g}$ is the radius of gyration associated with the microstructure size, and $G$ and $F$ are scale factors at low $Q$ and high $Q$, respectively. For 3D globular objects (such as spheres), $s=0$, for rods $s=1$ and for lamellae $s=2$. We note that similar values of $m$ are obtained if the Beaucage model is fitted. ${ }^{17}$

The fitted parameters are given in Table I. The Guinier exponents $s$ for ZM-MS4000-SPS400 and ZM-MS4000SPS500 samples are 2.35 and 2.69 ( \pm 0.01$)$, respectively. $2<s<3$ indicates that the microstructure is a network of lamellae in both samples. When the SPS temperature is increased, the value of $s$ increases. This means that a higher SPS temperature generates a higher density of lamellae network in the bulk samples. The thickness of the lamella in both samples is $\sim 10 \mathrm{~nm}$ (thickness $=\sqrt{12} R_{g}=17 \mathrm{~nm}$ and $12 \mathrm{~nm}$, for ZM-MS4000-SPS400 and ZM-MS4000-SPS500 samples, respectively). Both samples have a very similar value of $m \approx 3.8$ at high $Q$ within error. This indicates that they have microstructures with smooth interfaces, just as in the original ZM-ingot. Microstructures with a size of order

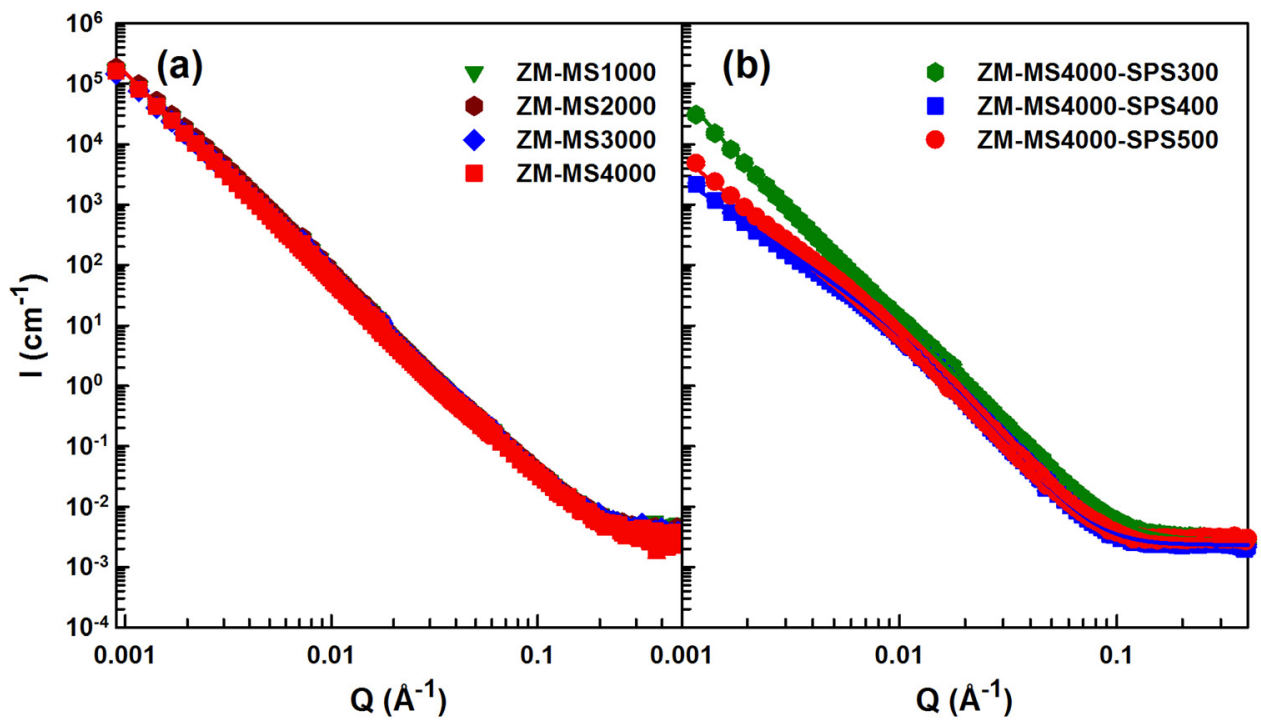

FIG. 2. Small-angle neutron scattering data of (a) the ZM-MSx samples as a function of rotation speed. All ZM-MSx samples follow the power-law. (b) The ZM-MS4000-SPSy samples as a function of SPS temperature. The ZMMS4000-SPS300 follows the power-law. The ZM-MS4000-SPS400 and ZMMS4000-SPS500 are fitted with the generalized Guinier-Porod model. The solid lines are fits to the data. 


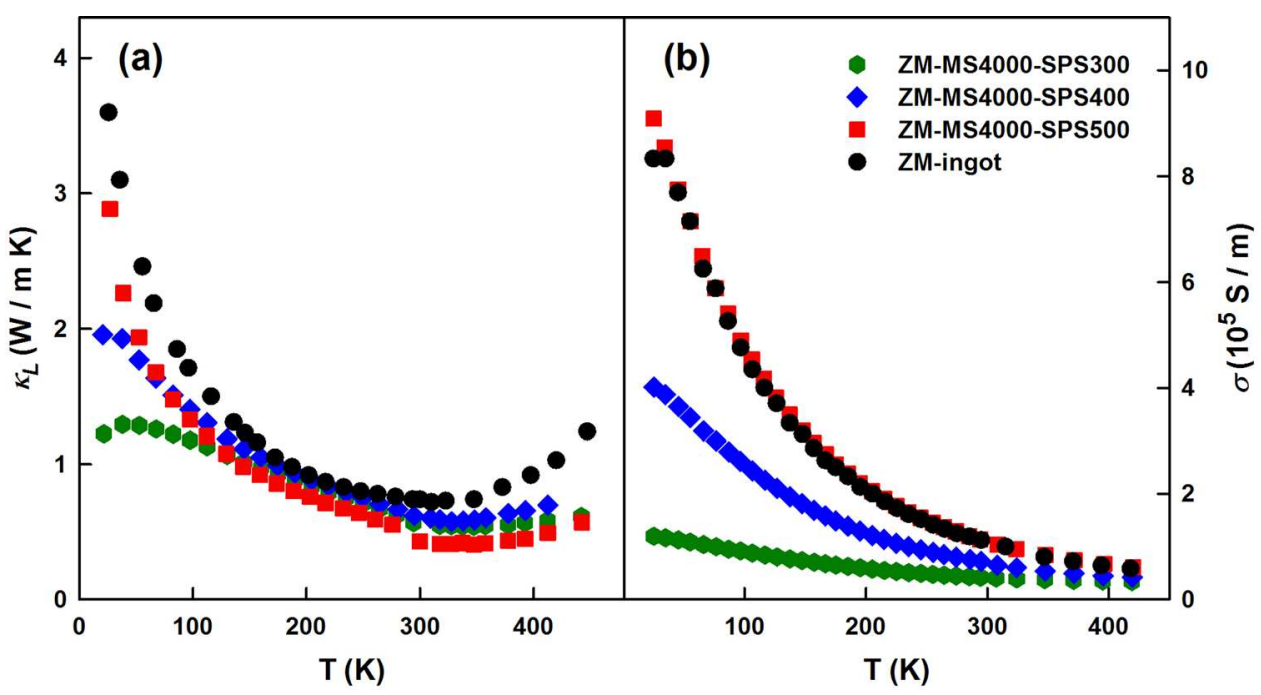

FIG. 3. Thermoelectric properties of ZM-ingot and ZM-MS4000-SPSy samples as a function of SPS temperature. (a) Lattice thermal conductivity. (b) Electrical conductivity. of $\sim 10 \mathrm{~nm}$ with smooth interfaces have been observed in $(\mathrm{Bi}, \mathrm{Sb})_{2} \mathrm{Te}_{3}$ samples in electron microscopic images, when they are synthesized by the MS-SPS process. ${ }^{12}$

To study the impact of the microstructures' interfaces and network on thermoelectric properties, we measured lattice thermal conductivity $\kappa_{l}$ and electrical conductivity $\sigma$ of the ZMMS4000-SPSy samples and the ZM-ingot. Fig. 3(a) shows that lattice thermal conductivity decreases in the ZM-MS4000SPSy samples compared to that in the ZM-ingot. This is due to the nanoscale microstructures generated by the MS-SPS process in the ZM-MS4000-SPSy (y=400, 500) samples and the rough interfaces of microstructures in the ZM-MS4000SPS300 sample. A rough interface could degrade electrical conductivity by scattering electrons at the boundaries. Indeed, Fig. 3(b) shows substantial reduction in electrical conductivity in the ZM-MS4000-SPS300 sample. However, electrical conductivity was increased in the ZM-MS4000-SPS400 and ZMMS4000-SPS500 samples. This can be explained by (1) the smoothing of the interfaces and (2) the networking of the nanoscale microstructures through the SPS process at high temperature $\left(>300^{\circ} \mathrm{C}\right)$, as suggested from our small-angle neutron scattering study. The highest ZT of 1.5 is achieved at $383 \mathrm{~K}$ in the ZM-MS4000-SPS500 sample. This value is very similar to that of samples in Refs. 11 and 12.

In summary, our finding is that the bulk $(\mathrm{Bi}, \mathrm{Sb})_{2} \mathrm{Te}_{3}$ samples with high thermoelectric efficiency have a microstructure network of $\sim 10 \mathrm{~nm}$ thick lamellae and smooth interfaces between them. Our small-angle neutron study shows (1) that the MS process generates multiscale microstructures with rough interfaces and (2) that the SPS process forms a nanoscale microstructure network with smooth interfaces. Comparing the physical properties, we find that rough interfaces of multiscale microstructures are responsible for the reduction of lattice thermal conductivity and electrical conductivity. The increase of electrical conductivity is achieved by the formation of a nanoscale microstructure network with smooth interfaces by the subsequent SPS process, which has little effect on lattice thermal conductivity. We also find that the smoother the interface and the higher the density of the nanoscale microstructure network, the better the electrical conductivity.

The work at Clemson University was supported by DOE/EPSCoR Implementation Grant No. DE-FG02-04ER46139 and the SC EPSCoR cost sharing program. The work at Wuhan University of Technology was supported by the National Basic Research Program of China (Grant No. 2007CB607501), the International Science and Technology Cooperation Program of China (Grant No. 2011DFB60150), and the 111 Project of China (Grant No. B07040). M.L. acknowledges support from Dan Scatt. The neutron scattering work at NIST was supported in part by the National Science Foundation under Agreement No. DMR-0454672.

${ }^{1}$ L. D. Hicks and M. S. Dresselhaus, Phys. Rev. B 47, 12727 (1993).

${ }^{2}$ T. C. Harman, P. J. Taylor, M. P. Walsh, and B. E. LaForge, Science 297, 2229 (2002).

${ }^{3}$ R. Venkatasubramanian, E. Siivola, T. Colpitts, and B. O'Quinn, Nature 413, 597 (2001).

${ }^{4}$ R. Yang and G. Chen, Phys. Rev. B 69, 195316 (2004).

${ }^{5}$ K. F. Hsu, S. Loo, F. Guo, W. Chen, J. S. Dyck, C. Uher, T. Hogan, E. K. Polychroniadis, and M. C. Kanatzidis, Science 303, 818 (2004).

${ }^{6}$ W. Kim, J. Zide, A. Gossard, D. Klenov, S. Stemmer, A. Shakouri, and A. Majumdar, Phys. Rev. Lett. 96, 045901 (2006).

${ }^{7}$ M. S. Dresselhaus, G. Chen, M. Y. Tang, R. G. Yang, H. Lee, D. Z. Wang, Z. F. Ren, J.-P. Fleurial, and P. Gogna, Adv. Mater. 19, 1043 (2007).

${ }^{8}$ R. Yang, G. Chen, and M. S. Dresselhaus, Phys. Rev. B 72, 125418 (2005).

${ }^{9}$ A. Majumdar, Science 303, 777 (2004).

${ }^{10}$ H. J. Goldsmid, Thermoelectronic Refrigeration (Plenum, 1964).

${ }^{11}$ B. Poudel, Q. Hao, Y. Ma, Y. Lan, A. Minnich, B. Yu, X. Yan, D. Wang, A. Muto, D. Vashaee, X. Chen, J. Liu, M. S. Dresselhaus, G. Chen, and Z. Ren, Science 320, 634 (2008).

${ }^{12}$ W. Xie, J. He, H. J. Kang, X. Tang, S. Zhu, M. Laver, S. Wang, J. R. D. Copley, C. M. Brown, Q. Zhang, and T. M. Tritt, Nano Lett. 10, 3283 (2010).

${ }^{13}$ H. D. Bale and P. W. Schmidt, Phys. Rev. Lett. 53, 596 (1984).

${ }^{14}$ B. Hammouda, J. Appl. Crystallogr. 43, 716 (2010).

${ }^{15}$ A. Guinier and G. Fournet, Small-Angle Scattering of X-Rays (John Wiley $\&$ Sons, New York, 1955).

${ }^{16} \mathrm{O}$. Glatter and O. Kratky, Small-Angle X-Ray Scattering (Academic, London, 1982).

${ }^{17}$ G. Beaucage, H. K. Kammler, and S. E. Pratsinis, J. Appl. Cryst. 37, 523 (2004). 